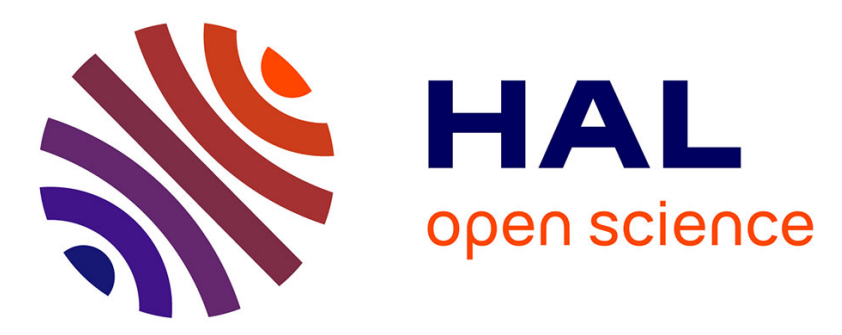

\title{
The CPS and LCA Modelling: An Integrated Approach in the Environmental Sustainability Perspective
}

Andrea Ballarino, Carlo Brondi, Alessandro Brusaferri, Guido Chizzoli

\section{To cite this version:}

Andrea Ballarino, Carlo Brondi, Alessandro Brusaferri, Guido Chizzoli. The CPS and LCA Modelling: An Integrated Approach in the Environmental Sustainability Perspective. 18th Working Conference on Virtual Enterprises (PROVE), Sep 2017, Vicenza, Italy. pp.543-552, 10.1007/978-3-319-65151-4_48 . hal-01674859

\section{HAL Id: hal-01674859 \\ https://hal.inria.fr/hal-01674859}

Submitted on 3 Jan 2018

HAL is a multi-disciplinary open access archive for the deposit and dissemination of scientific research documents, whether they are published or not. The documents may come from teaching and research institutions in France or abroad, or from public or private research centers.
L'archive ouverte pluridisciplinaire HAL, est destinée au dépôt et à la diffusion de documents scientifiques de niveau recherche, publiés ou non, émanant des établissements d'enseignement et de recherche français ou étrangers, des laboratoires publics ou privés. 


\title{
The CPS and LCA Modelling: An Integrated Approach in the Environmental Sustainability Perspective
}

\author{
Andrea Ballarino, Carlo Brondi*, Alessandro Brusaferri and Guido \\ Chizzoli \\ ITIA-CNR Institute for Industrial Technologies and Automation -, 12 Alfonso \\ Corti, 20133 Milan, Italy \\ \{andrea.ballarino, carlo.brondi, alessandro.brusaferri, \\ guido.chizzoli\}@itia.cnr.it
}

\begin{abstract}
The present paper clarifies the methodological framework to implement the integration of Cyber Physical Systems (CPS) in the perspective of environmental sustainability. Firstly, authors identify main methodological, software and hardware pre-requisites enabling the use of CPS systems in accordance with the literature and environmental standards. Then, the paper focuses on the main barriers in the case the information is distributed along a flexible supply chain and identifies a methodology to coordinate environmental information from ubiquitous sources by the use of modularization. This methodology is applied in the field of energy management for the steel sector for different production lines. In particular, it is highlighted as the consumption tracking may be linked to the real-time management of suppliers and processing cycles in order to pursue strategies for environmental impact minimization. Results emphasized as the development of the CPS systems that are designed for sustainability can produce relevant results for the effective restrain of industrial environmental impacts.
\end{abstract}

Keywords: CPS · LCA · Industrial Sustainability · Modular LCA · Industry 4.0 .

\section{Application of CPS for Environmental Sustainability}

The Cyber Physical Systems (CPS) are identified by many authors as key technology for the development of manufacturing industry in the next future [1] [2] [3] [4]. Such technology in fact introduces a number of significant potential for production systems such as the ability to process a large amount of data in real time, the ability to optimize operations by flexible and automatic solutions, and finally the ability to enhance collaboration between different levels of the factory and suppliers.

In particular, some authors identify the features that are obtained through the CPS systems such as a prodromal condition for the actual sustainability of production systems [3] [4] [5] [6]. Some authors emphasize also that the development of the CPS systems could lead further barriers to sustainability by massive production of devices 
and by causing significant social effects on employment market [3]. Despite such remarks, various beneficial contributions to sustainable manufacturing paradigm can be identified.

In the first place, the support of the CPS systems to Sustainable manufacturing can be performed through new instruments for sustainability policies. The Sustainable Manufacturing in fact requires the implementation of strategic objectives such as zero waste, the benign emissions, the use of renewable resources, the circular economy, the process efficiency, social investment, and the modification of trade criteria goods. Each of these objectives entails increasing complexity, operation intelligence and a growing integration between the operations along the supply chain and the facility [7] [8] [9]. The development of the CPS majorly affects these areas by increasing monitoring, collaboration and efficiency across several areas [1] [3] while in other cases the CPS constitutes the bulk of new sustainability-oriented technologies [6].

The second contribution of CPS regards the building of an infrastructure to ease the calculation of Key Performance Indicators (KPIs) for the sustainability of industrial practices. The environmental impact assessment of production operations, in fact, involves interdisciplinary areas and requires a data-intensive process. Such assessment increases in complexity with the concurrent increase of the production constrains. The development of CPS allows to reduce this convolution in calculation by capturing latent properties of ubiquitous systems. Furthermore, the independent behavior of CPS also enables to manage this exponential complexity in relation to response actions and decisional support [4]. Through such support, CPS makes possible to develop integrated environmental management systems that are constantly connected to the analytical values and environmental accounting and create a continuous stream of verifiable environmental information.

From another point of view, the implementation of CPS and industry paradigm 4.0 introduces a series of constraints that are needed to fully implement this potential capability. The main involved issues seem to concern the information role, its transfer, and the complexity management [5] as inevitable problems to be treated. Another important aspect is the reliability of data that are collected through sensor networks as well as their reuse [10] [11] [12].

CPS applications for sustainable manufacturing, by literature, include applications for circular economy in the modelling of Industrial Park [13], green computing to increase efficiency in energy consumption [1] [14] and other [6] applications.

\section{Requirements for the LCA Integration within the CPS Framework}

The intended purpose of such work is to sequentially clarify pre-requisites, current barriers and potentialities for LCA integration within CPS. The use of CPS in the environmental perspective, may allow a real time quantification of the environmental impact of a set of productive activities that are subject to a certain variability. From one side, this usage enables most reliable Life Cycle Assessment (LCA) calculation in comparison to traditional ex-post LCAs. According to CPS framework, LCA seems a suitable methodology to be integrated within CPS to quantify environmental 
implication of a certain activity by virtue of certain characteristics: LCA is designed to provide quantitative data; LCA is directly connected to business areas that monitor the business flows and which provide monitoring data for Life Cycle Inventory (LCI) phase; taking a perspective on the entire life cycle, the LCA methodology manage data that are external to company boundaries; finally, the LCA methodology is well supported in standardization by various ISO standards in the international arena, and then the generation and verification of the environmental information tend to be more congruent between different countries.

The expected progression CPS functions is characterized by five hierarchical levels [15], each level corresponds to a stage of CPS functional development. Equally, it is possible to define a progression of functions for real-time analysis of environmental impact that can be linked with the LCA. Each of these layers includes a number of prerequisites regarding the different elements involved, such as the sensors, the data management systems, the simulation methods, and the standardization and optimization criteria (fig. 1).

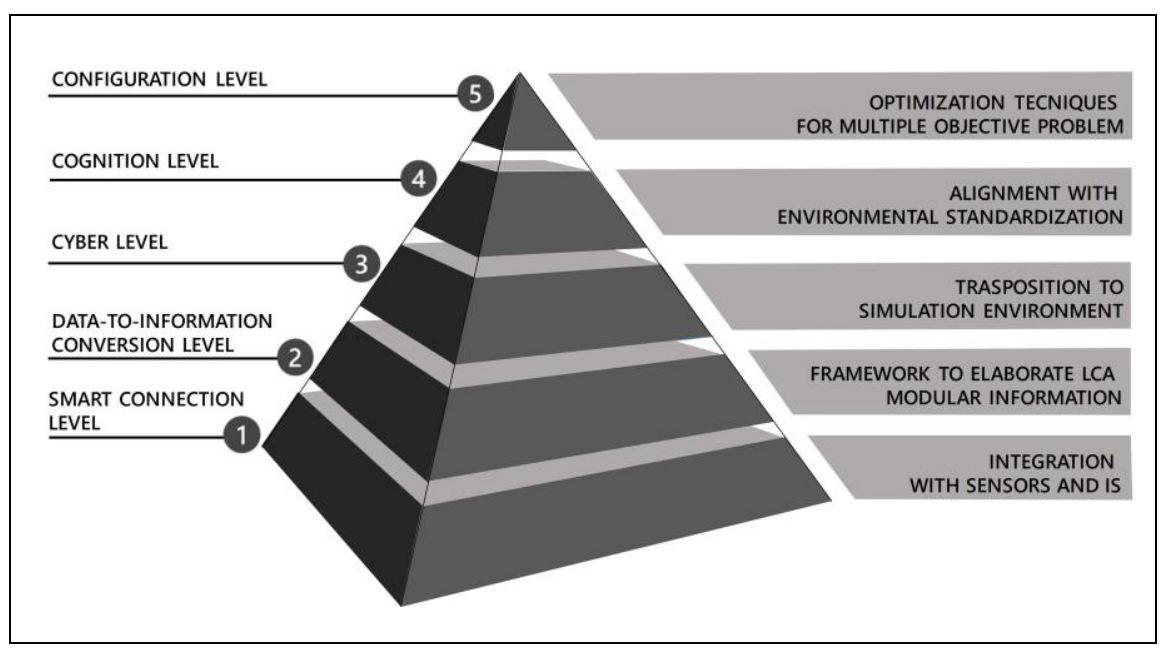

Fig. 1. Requirements for CPS use in the LCA framework

Smart connection level. At this level, the challenge to create a reliable LCA from distributed data depends on the chance to record data on the environmental aspects and to bring them to a higher level.

Therefore, it is necessary that CPS glean data from a distributed network of data sources regarding direct tracking trough sensors or indirect tracking through Information Systems (IS). Such data should be attributed to local mass and energy balances for a set of activities. The data collection system should track consumption of materials, energy or items as well as solid, liquid or gaseous emissions. The sequence of data collection assumes different trends depending on the characteristics of the flow to be monitored. 
As an example, the tracking of a homogeneous gaseous flow over the time will require an instantaneous flow rate measurement system while in the case the composition changes over time an extra measurement device is needed (e.g. a mass spectrograph) in order to identify the chemical composition. The data used by CPS systems is not necessarily derived from field sensors but can also be obtained through IS such as Enterprise Resource Planning (ERP) or Supply Chain Management (SCM) tools. CPS should recognize and capture these data from remote systems through the use of complementary information that must travel together to the data on physical features. In this sense, methodologies such as the semantic web could support the identification of standard protocols for data transmission. The data collection system also influences the temporal trend of its tracking (e.g. solid waste can be monitored on a monthly basis while electric power consumption can be monitored on a shorter basis). It would therefore be important to understand through a preventive analysis which are the critical flows and the related measurement policies.

Another key issue concerns the monitoring cost. The calculation of a time-variant mass/energy balance can be expensive for a company. Therefore, the development of "smart inventory" systems allows to produce more information through a single measure or a measures combination. Such indirect measurement can require inference methods and data combination in order to extrapolate the investigated environmental aspects.

Data-to-information connection level. At this level, the data collected from the field are transformed into meaningful information in environmental terms. As an example, it becomes possible to trace the carbon dioxide produced by a single task.

In terms of LCA this kind of transposition requires that the local impact is defined through a modification of traditional LCA calculation criteria. The Goal and Scope phases, for example, can become reconfigurable at an upper level depending on the end-user needs. The information storage becomes no longer a remote activity, instead the information is continuously available. Such a transformation requires a modularization of environmental information that no longer refers to the entire life cycle but can be considered as congruent assembly of gate-to-gate modules.

The modular approach has already been applied in the manufacturing environment [16]. A second important aspect concerns the final assessment quality that must be suitable for a major precision in comparison to traditional LCAs.

Data to be processed should be attributable to the same functional units and should be supplied with standard information to support upper level calculation as allocation and quality assessment. As a fundamental requirement, the application of the LCA modularization requires connecting the material and energy flow monitoring, namely the inventory of the environmental aspects, with the environmental profile of individual flows. Therefore data are associated with database that are external to the physical CPS. The databases roles include both data storing for long-term strategies and active capabilities in supporting CPS to govern the production systems operations [5]. These stored data, which are updated by a different rate in comparison to physical flow data, requires also an intermediate step for respective association in order to be referred to the same activity (e.g. an absorbed current should be referred both to a certain operation and to an incoming energy flow from a specific supplier).

Cyber level. At this level, it becomes possible to express the aggregate environmental impact and to simulate the LCA-based environmental efficiency of the 
CPS through the mirroring of the behaviour of a production unit in a virtual environment. The CPS should express a function for instantaneous and predictive impact on the environment for the cumulated activities under its control. Simulation is the essential step to perform such activity. In environmental terms, environmental profile function can be calculated as modular variable environmental profile constituted by the instantaneous value of the different impact categories.

At this level, the CPS also coordinates with other analogous units to exchange information on other activities that have influence on its environmental impact. Also in this case, the communication should be supported by protocols aiming to coordinate and address information. Such aspect concerns the automatization of LCA procedures such as allocation of field measurements and information alignment. The CPS unit should report together with the final data, information on the overall quality of the calculation, complementary information on calculation criteria (allocations, the system boundaries, geography, period sampling etc.). In the absence of these complementary information, the environmental impact function risk to be inferred by incongruent modules leading to a worse result than the traditional LCA. Some examples of protocols for standardization of environmentally-related information has been studied for the UML language for the Manufacturing Execution System (MES) [17] and the Product Life Cycle Management (PLM) [18].

In terms of simulation criteria, different criteria can be linked to LCA. Discrete event simulation [19] [20] has been applied to model the environmental impact for different production scenarios. According to these criteria, modelling of the behaviour of a production unit is separated from its actual field operation, the same impact tends to depend on general purpose database.

The application of a simulation that is connected with the real time operational value can introduce a direct link with industry-driven data within simulation both in term of inventory data and performed assessment. In addition, the introduction of dynamic simulation methods allows to extend the LCA beyond its normal scope of and to create the basis for the application of the optimization criteria.

On the other hand, the creation of a CPS "virtual twin" requires the application of calibration methods. An initial calibration relates to the explicit link between environmental drivers and environmental effects within the simulation model. The LCA methodology is based on "black box" structure while the simulation requires a certain degree of explication for the links between the environmental drivers and their effects according to a logic of "grey box". In this sense, the modularization should be designed in such a way that the information might be cut out in the perspective use for the simulation. A second type of calibration regards the temporal correlation between the field measurements and simulation of an activity. If the sampling of the mass flows and energy are delayed in time with respect to the range of the simulation calculation system may inherit an implicit inaccuracy.

As an example, a certain input value function (e.g. a function for a processing material) may report constant value between two sampling moments while other input values (e.g. compressed air functions) are varying independently from the source behaviour. It appears therefore necessary to partially align the sampling times and simulation times. Finally, the time trend of some driver value that comes from remote 
sources can be unpredictable. It will be necessary therefore to use regression methods to reduce uncertainty in "time machine" simulation.

Cognition level. At this level data from CPS level are further elaborated in order to provide key performance indicators that are compliant with existing environmental standards. At this level, it is also possible to link CPS data with other sustainability accounting systems (e.g. cost accounting or social accounting) or company accounting system (fig. 2). The existence of misaligned industrial simulation areas can limit the transition from an accounting system to another due to the fact such areas are still not integrated (e.g. product design, process simulation, ergonomics, etc.). Further integration of simulation areas can create a holistic simulation area that affects different decision areas. In such sense, knowledge domain integration performed by ISA 95 and other standards can also foster CPS development.

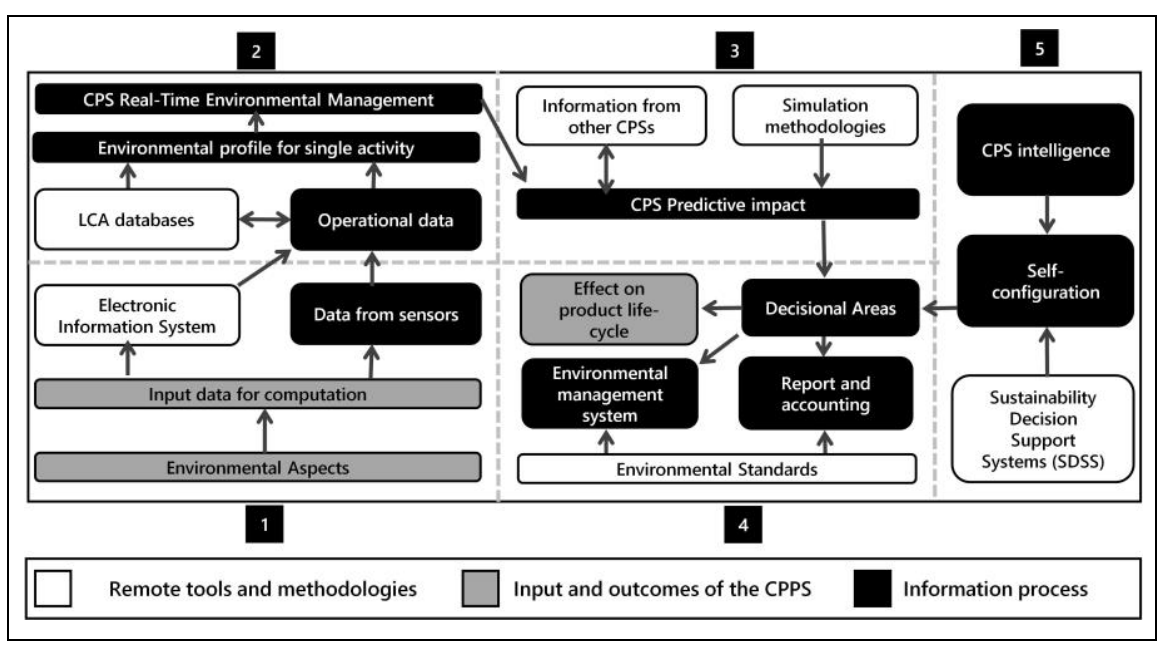

Fig. 2. Data elaboration in the environmental perspective through different CPS levels

In terms of environmental performance CPSs can pave the way to new generation of Environmental Management Systems (EMS) within companies through the creation of a continuous digitized assessment of environmental information. The modular and flexible structure of environmental data from CPSs should allow to quantify the environmental performance of different business areas as well as of different stages of the product life cycle.

Configuration level. At this last stage, CPS can self-configure itself based on real time LCA outcomes, system constraints and Objective Functions (OF) related to sustainability targets or other targets. The proper development of this function requires that environmental optimization criteria are clearly addressed. LCA can be already applied for process optimization according to an environmental sustainability perspective. The general problem for optimization has a multi-objective nature cause to the distinctive features of the LCA characterization indicators, the key performance representation according to international standards, and the perspective integration of these indicators with other non-environmental performance indicators. In 
mathematical terms, the branch of the Sustainability Decision Support System includes a range of analytical support methods that are based on artificial intelligence (Expert System-ES, Genetic Algorithm-GA, Artificial Neural, Network-ANN, Intelligent Agents- IA, Case Based Reasoning-CBR), but also other Geographic Information System-GIS-based methodologies, Internet-based services, and Business Intelligence BI. In the presence of different criteria and priorities, effective automatic self-configuration by the CPSs exist in case multi-objective optimization system is transparently and congruently addressed.

\section{Application of CPS to Energy Grids for LCA based Optimization}

The potential application of CPS systems has been explored in the case of an energy supply system for a European process industry company. The application of CPS framework was implemented in a smart grid in order to manage real-time data to optimize energy consumption in the sustainability perspective from the point of view of the final consumer industry. These CPSs can exchange real-time information on energy attributes and real-time demand in a coordinate way (fig.3).

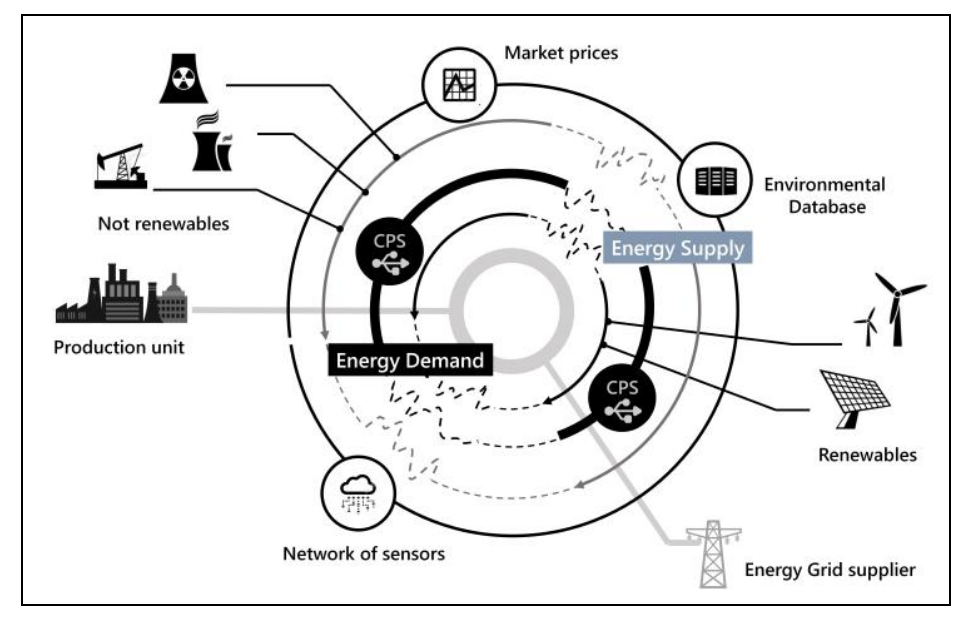

Fig. 3. Application of CPS for Environmental optimization $\backslash$ of energy grid

The user company has already implemented a system of sensors that can record energy consumption from electrical grid and the CPS system is able to estimate the energy consumed for each work cycle and, prospectively, the energy required for subsequent scheduling through a Time-machine system. User can gain more insight on the system variation and self-adjust consumption according to prediction of everchanging attributes of energy production pattern (costs, environmental impacts etc.).

Similarly, the energy supplier is able to transfer a series of real-time information regarding the average hourly energy price and, at the same time, the LCA-based environmental information impact. Both information are based on regression analysis 
when CPS simulate the future energy supply. Information on price is based on opensource database for energy market while the Environmental impact is based on combined information by the national energy authority on the actual allocation between renewable energy and fossil-based energy production. The system thus incorporates the ideal features for the implementation of CPS systems since the monitoring of environmental aspects (energy consumption) and energy consumptions present performance that are time dependent. In this case, the CPS allows to represent the environmental performance through equation 1 :

$$
\boldsymbol{I}_{w}(t+1)=A \cdot \int_{t}^{t+1} \boldsymbol{k}(t) * w(t)
$$

In such equation, $\boldsymbol{I}$ represents the energy performance indicator at a given moment $\mathrm{t}+1$ for a number of monitored operations, $\boldsymbol{k}(t)$ represents the vector of unit flow performance, $\mathrm{w}(\mathrm{t})$ represents the instantaneous absorbed power by the company at the moment $\mathrm{t}$ for the monitored operations while $\mathrm{A}$ is a normalization coefficient.

The variable $\mathbf{k}(\mathrm{t})$ depends on the monitoring of the CPS on energy network conditions and is partly based on the LCA databases support, while $w(t)$ depends on the instantaneous monitoring of consumption within the company through sensor system.

$$
\boldsymbol{k}_{\boldsymbol{e}}(t)=\sum_{s=1}^{S} \frac{\boldsymbol{k}_{\boldsymbol{s}}(t) \cdot p_{S}(t)}{P_{\text {tot }}(t)}
$$

In equation $2, \mathbf{k}_{\mathbf{e}}(\mathrm{t})$ represent the environmental impact vector for the supply of energy at time $\mathrm{t}$ from the network. $\boldsymbol{k}_{\boldsymbol{s}}(\mathrm{t})$ corresponds to the unitary energy impact vector for a given energy source $s$ for the total of S energy production technologies (e.g. carbon dioxide equivalent for one kwh that is produced by solar) while $p_{s}$ and $P_{t o t}$ represent respectively the provided energy power on the energy grid at instant $t$ by using the energy generation technology $s$ and the total energy power available on the grid.

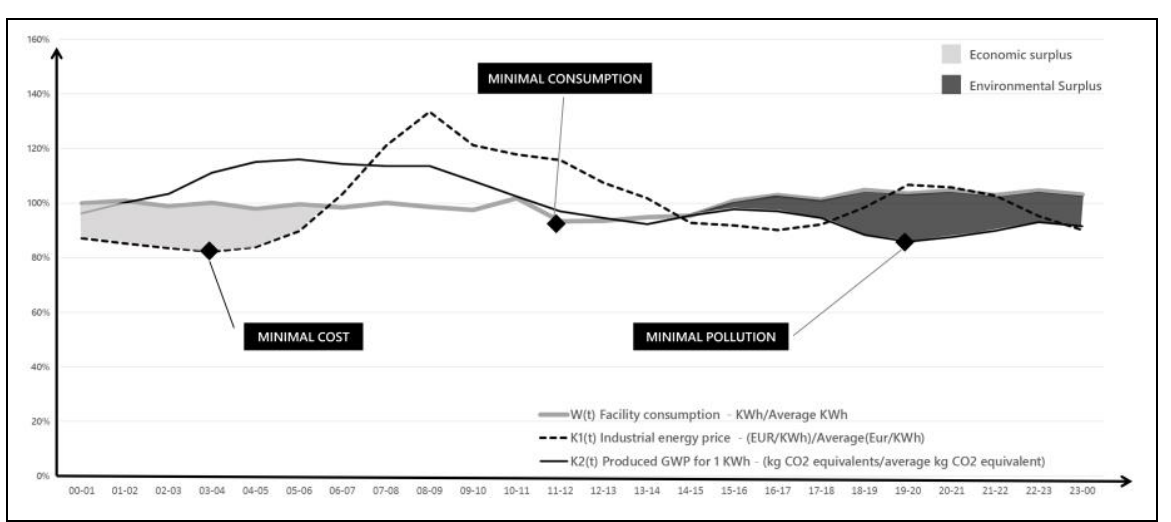

Fig. 4. Daily regression energy analysis according to CPS real-time monitoring system

The objective function to be optimized in this case coincides with a multi-objective function that concurrently minimizes $I_{w}$ as a set of indicators. Outcome for such 
applications regards the identification of time spans for focusing production in correspondence of minimal energy cost or minimal environmental impact. Sustainability profile resulting from operations arrangement of the examined factory can be improved simply by introducing time constrains to single operations. Open issues still remain in identifying methodologies for concurrent optimization of cost and environment that are not based on arbitrary weighting of such categories. At the same time, the formalization of production constrains in the optimization model could better emphasize margins for green manufacturing policies.

\section{Conclusion and Remarks}

The implementation of CPS can have positive and remarkable effect for the implementation of Sustainable Manufacturing policies. In particular, the implementation of LCA tools can introduce a quantitative basis to provide effective environmental improvements in virtue of its intrinsic characteristic. The proper implementation of CPS systems integrating LCA features can concurrently provide real-time management systems integrating factory and supply chain level, efficient EMS and provide effective tools to adapt operational management to high-level sustainability targets. Such progresses introduce the possibility of intelligent production unit that are able to adjust and record their environmental impact. CPS can in fact include the ubiquity of information in the production chain and manage the complexity of the criteria to calculate the environmental impact. However, its implementation poses a number of challenges and constrains to industry automation at semantic, physical, operational and decisional levels. In particular, protocol development for data integration appears as an indispensable key area to foster CPS application for eco-efficiency. Apparently, the use of CPS seems to imply major effects in presence of certain operational characteristics as the congruency between variance of different operational drivers, the cost and availability of field data and margins for company to address such indications within decisional areas. According to the presented case, the application of CPS to smart energy grids seems to meet all these features. Improvements in real-time and foresight LCA assessments introduce measurable improvements on specific sustainability target. Perspective work can be oriented on the further formalization of production constrains as well as to reliability of regression analysis by a comparison of an ex-ante optimization policies and ex-post gate-to-gate analysis of effective environmental impacts. In parallel, further research on multi-criteria optimization can enforce CPS intelligence for such purpose.

\section{References}

1. Schuh G., Reuter C., Hauptvogel A.: Increasing collaboration productivity for sustainable production systems. Procedia CIRP 29, 191-196 (2015)

2. Siemieniuch C.E., Sinclair M.A., Henshaw, M.J.DeC.: Global Drivers, Sustainable Manufacturing and system ergonomics. Applied Ergonomics 51, 104-119 (2015) 
3. Waibel M.W., Steenkamp L.P., Moloko N., Oosthuizen G.A.: Investigating the effects of Smart Production Systems on sustainability elements. In: 14th Global Conference on Sustainable Manufacturing, GCSM 3-5 October 2016, Stellenbosch, South Africa. Procedia Manufacturing 8, 731-737 ( 2017 )

4. Dumitrache I., Caramihai S. I.: The Enterprise of Future as a Cyber-Physical System. In: 7th IFAC Conference on Manufacturing Modelling, Management, and Control June 19-21. Saint Petersburg, Russia pp. 1310-1315 (2013)

5. Bonci A., Pirani M., Longhi S.: A database-centric approach for the modeling, simulation and control of cyber-physiscal system in the factory of the future. In: IFAC-PapersOnLine 49-12, 249-254 (2016)

6. Estevez C., Wu J.: Green Cyber-Physical systems. In: Cyber-Physical Systems, Chapter 15, Elsevier (2017)

7. Esmaeilian B., Behdad S., Wang B.: The evolution and future of manufacturing: A review. Journal of Manufacturing Systems. 39, 79-100 (2016)

8. Zhang H, Calvo-Amodio J, Haapala KR. A conceptual model for assistingsustainable manufacturing through system dynamics. Journal of Manufactur-ing Systems;32(4), 543-9 (2013).

9. Haapala KR, Zhao F, Camelio J, Sutherland JW, Skerlos SJ, Dornfeld DA, et al.A review of engineering research in sustainable manufacturing. J Manuf SciEng 135(Jul (4)), (2013)

10.Tang L., Yu X., Kim S., Gu Q., Hana J., Leung A., La Porta T.: Trustworthiness analysis of sensor data in cyber-physical systems. Journal of Computer and System Sciences 79, 383$401(2013)$

11.Wang D.: Data reliability challenge of cyber-physical systems. In: Cyber-Physical Systems, Chapter 6, Elsevier (2017)

12.DeSmit Z., Elhabashy A. E., Weels L.J., Camelio J. A.: An approach to cyber-physical vulnerability assessment for intelligent manufacturing systems. Journal of Manufacturing Systems (in press) (2017)

13.Pan M., Sikorski J., Kastner C. A., Akroyd J., Mosbach S., Lau R., Kraft M. : Applying Industry 4.0 to the Jurong Island Eco-industrial Park. Energy Procedia 75, 1536-1541 (2015)

14.Gupta S.K.S., Mukherjee T., Varsamopoulos G., Banerjee A.: Research directions in energy-sustainable cyber-physical systems. Sustainable Computing: Informatics and Systems 1, 57-74 (2011)

15.Kao H. A. , Jin W., Siegel D., Lee J.: A Cyber Physical Interface for automation SystemsMethodology and Examples. Machines 3, 93-106 (2015)

16.Brondi C., Carpanzano E.: A modular Framework for the LCA-Based simulation of production systems. CIRP Journal of Manufacturing Science and Technology 4, 305-312 (2011)

17.Lee H., Ryu K., Son Y.J., Cho Y.: Capturing Green Information and Mapping with MES Functions for Increasing Manufacturing Sustainability. International Journal of precision Engineering and Manufacturing 15/8, 1709-1716 (2014)

18.Zhao, W.B., Park Y.H., Lee H. Y., Jun C. M., Noh S. D.: Design and Implementation of a PLM System for Sustainable Manufacturing. In: Rivest L., Bouras A., Louchichi B.(Eds): PLM 2012, IFIP AICT 388, pp.202-212, (2012).

19.Logfren B., Tillman A.: Relating manufacturing system configuration to life-cycle environmental performance: discrete-event simulation supplemented with LCA. Journal of Cleaner Production 19, 2015-2024 (2011)

20.Sproedt A., Plehn J., Schonsleben P., Hermann C.: A simulation-based decision support for eco-efficiency improvements in production systems. Journal of Cleaner Production 105, $389-405(2015)$ 